\section{Integrative Medicine \\ International}

Integr Med Int 2014;1:170-175

\title{
Constitutional Study of Parkinson's Disease Patients Based on Traditional Chinese Medicine Pattern Differentiation
}

\author{
Jing-Jing Li Ka-Kit Chua Min Li \\ School of Chinese Medicine, Hong Kong Baptist University, Hong Kong, SAR, China
}

\begin{abstract}
Key Words
Parkinson's disease - Constitutions - Traditional Chinese medicine - Pattern differentiation . Levodopa
\end{abstract}

\begin{abstract}
Objectives: It was the aim of this study to determine whether (1) Parkinson's disease (PD) patients can be divided into different traditional Chinese medicine (TCM) constitutions based on the 44-item Traditional Chinese Medical Constitutional Scale and Classification (TCM-CSC, Chinese version, 中醫體質及分型量表)，(2) whether levodopa usage correlates with the TCM classification type, and (3) whether there is a difference in the mean daily intake of levodopa between males and females in any of the constitutional types. Methods: PD patients who had visited the TCM clinic of the Hong Kong Baptist University (HKBU) or who had attended the PD public talks organized by HKBU and the Association of Parkinson's Disease in Hong Kong were invited for interviews with TCM assessors. The TCM-CSC (Chinese version) was used in the assessment to divide patients into four different constitutions: the balanced constitution (type A), the yang-deficiency constitution (type B), the yin-deficiency constitution (type C) and the stasis constitution (type D). Results: Sixty-three patients (29 males, 34 females; mean age $63.65 \pm 8.116$ years; mean PD duration $5.49 \pm 4.544$ years) participated in the study. Sixteen PD patients (26\%) had only one type of TCM constitution, while 47 PD patients (74\%) had more than one type of TCM constitution. Type D patients showed the lowest intake of daily levodopa. There was no significant difference in the mean daily intake of levodopa between males and females in any of the constitutional types. Conclusions: (1) PD patients cannot be divided into different constitutional types based on the TCM-CSC effectively. (2) In general, levodopa usage does not correlate with the constitutional type; however, patients with the sta-
\end{abstract}

J.-J.L. and K.-K.C. contributed equally to this study. 
sis constitution (type D) showed the lowest levodopa intake compared to the other constitutions. (3) There was no significant difference in the mean daily intake of levodopa between males and females. Further research on the treatment pattern of different TCM constitutions, using large samples, is warranted.

(C) 2015 S. Karger AG, Basel

\section{Introduction}

Parkinson's disease (PD) is the second most common neurodegenerative disease in the world, with a prevalence rate of $1 \%$ in the population over the age of 60 years [1]. It is a heterogeneous disease both clinically and pathologically [2], including motor and non-motor symptoms [3]. Resting tremor, rigidity, bradykinesia and postural and gait impairment are the four major motor clinical features [4]; mood disorders, gastrointestinal disturbance, pain and sleep disorders are some common non-motor clinical features [5]. Attempts have been made to differentiate PD into subtypes for treatment purposes; however, no classification system has been adopted, as no system has been able to consistently indicate a more effective treatment for subtypes.

In the past, in Western medicine, PD was divided into subtypes based on motor or nonmotor symptoms [6]. In traditional Chinese medicine (TCM), patients are divided into different constitutional types based on their TCM diagnosis [7]. There is little study on the constitutions of PD patients in TCM. In our study, we investigated the existence as well as the usage of levodopa in the different TCM constitutions of PD patients by using a validated TCM classification questionnaire. We also assessed differences in the usage of levodopa by gender. Our objectives were to determine (1) whether differentiating PD patients by TCM classification was possible, (2) whether levodopa usage correlated with the TCM classification type, and (3) whether levodopa usage correlated with gender, particularly within TCM classification types. We hope that our results will provide a new, productive direction for further research in the treatment of PD.

\section{Subjects and Methods}

Between February and April 2014, 72 PD patients who had visited the TCM clinic of the Hong Kong Baptist University (HKBU) or who had attended the PD public talks organized by HKBU and the Association of Parkinson's Disease in Hong Kong (HKPDA) were invited for interviews with TCM assessors. PD patients were excluded if they did not agree to be interviewed. All TCM assessors had been well trained in a preliminary workshop to ensure consistency during the interviews.

The questionnaire used was the 44-item Traditional Chinese Medical Constitutional Scale and Classification (TCM-CSC, Chinese version, 中醫體質及分型量表), which was developed by Lin and colleagues [8-12] from Taiwan, to identify four constitutions: type A: the balanced constitution (平和質); type B: the yang-deficiency constitution (陽虛質); type C: the yin-deficiency constitution (陰血虛質), and type D: the stasis constitution (疮滯質).

The questionnaire comprises a total of 44 questions, of which 19 are related to type B, 19 to type $\mathrm{C}$ and 16 to type D. For the type $\mathrm{B}$ questions, a total score of $\geq 30.5$ indicates that the person is of the yang-deficiency constitution $[8,9]$. For the type $C$ questions, a total score of $\geq 29.5$ indicates that the person is of the yin-deficiency constitution $[10,11]$. Similarly, for the type $\mathrm{D}$ questions, the cutoff score is 26.5 [12]. Patients who score above the cutoffs on more than one type belong to multiple types, type $\mathrm{B}+\mathrm{C}$, type $\mathrm{B}+\mathrm{D}$, type $\mathrm{C}+\mathrm{D}$ and type $\mathrm{B}+\mathrm{C}+\mathrm{D}$, 
Li et al.: Constitutional Study of Parkinson's Disease Patients Based on Traditional Chinese Medicine Pattern Differentiation

Fig. 1. Percentage distribution of PD constitution by the TCM-CSC. Sixteen patients had only one type (white section), 16 patients had 2 types (light grey section) and 31 patients had 3 types (dark grey section) of constitution.

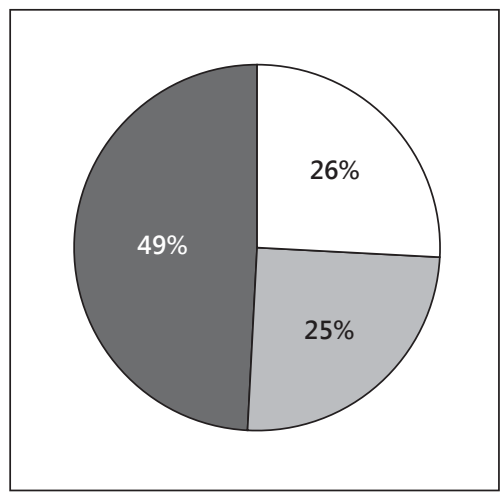

Table 1. Basic characteristics of PD patients

\begin{tabular}{ll} 
Age, years & $63.65 \pm 8.12$ \\
Patients & 63 \\
$\quad$ Females & 34 \\
$\quad$ Males & 29 \\
PD duration, years & $5.49 \pm 4.54$ \\
$\quad$ Males & $5.69 \pm 4.89$ \\
$\quad$ Females & $5.32 \pm 4.67$ \\
\hline
\end{tabular}

Values are given as the mean \pm SD or number of patients.

accordingly. Finally, patients who score below all cutoff points are assigned to type A. All data were collected by J. J. Li and checked by K. K. Chua. Analyses were done with SPSS 20.0 package (SPSS, Chicago, Ill., USA).

According to the questionnaire results, patients were divided into 6 groups: type A, type $B$, type C, type D, dual (i.e., patients who scored above the cutoff points of either two types of B, C or D) and triple types (i.e., patients who scored above the cutoff points for types A, B and D). Based on these results, analyses were carried out to determine if there was any correlation between each constitution and daily levodopa intake. Further analyses were done to determine any differences in daily levodopa intake for different genders within each constitutional type.

\section{Results}

Seventy-two PD patients agreed to be interviewed; 63 completed the interview. Demographic data and clinical characteristics of these 63 participants are summarized in table 1. The 63 patients included 29 males and 34 females. The mean age of all participants was 63.65 \pm 8.12 years, and PD duration in patients was $5.49 \pm 4.54$ years. 16 PD patients $(26 \%)$ had only one type of TCM constitution, of whom $11(17.46 \%$ of the total or nearly half of the singleconstitution group) were in the balanced constitution group (type A), $1(1.59 \%)$ had a yangdeficiency constitution (type B), 2 (3.17\%) had a yin-deficiency constitution (type C), and 2 $(3.17 \%)$ had a stasis constitution (type D). Most of the patients interviewed (47, 74\%) had more than one type of TCM constitution (fig. 1), and of these, 31 PD patients scored above the cutoff points for all three constitutional types (type B + C + D). As PD patients may fall into the type A group if they failed to be classified into 1 of the other 3 groups, no one had the combination type with type $A$. In other words, type $A$ (11 patients) and type B $+C+D(47$ patients) 
Li et al.: Constitutional Study of Parkinson's Disease Patients Based on Traditional Chinese Medicine Pattern Differentiation

Table 2. Number of patients in each constitution

\begin{tabular}{lccl}
\hline Constitution & Patients & $\begin{array}{l}\text { Daily levodopa } \\
\text { intake, mg }\end{array}$ & $\begin{array}{l}\text { PD duration, } \\
\text { years }\end{array}$ \\
\hline Type A & 11 & $213.64 \pm 256.02$ & $5.18 \pm 4.62$ \\
Type B & 1 & $400.00 \pm 0$ & $1.00 \pm 0$ \\
Type C & 2 & $375.00 \pm 530.33$ & $7.50 \pm 0.71$ \\
Type D & 2 & 0 & $7.50 \pm 7.78$ \\
Type B + C & 6 & $133.33 \pm 147.20$ & $3.00 \pm 1.67$ \\
Type B + D & 6 & $50.00 \pm 122.47$ & $4.50 \pm 5.47$ \\
Type C + D & 4 & $87.50 \pm 143.61$ & $9.25 \pm 6.85$ \\
Type B + C + D & 31 & $295.16 \pm 488.60$ & $5.68 \pm 4.60$ \\
\hline
\end{tabular}

Values are given as the mean \pm SD or number of patients.

Table 3. Daily levodopa intake of males and females in different constitutions

\begin{tabular}{llcccl}
\hline Constitution & Females & Levodopa intake, $\mathrm{mg} / \mathrm{day}$ & Males & Levodopa intake, mg/day & $\mathrm{p}$ value \\
\hline Type A & 8 & $183.33 \pm 160.73$ & 3 & $225.00 \pm 292.77$ & 0.773 \\
Type B & 1 & $400 \pm 0$ & 0 & N $/ \mathrm{A}$ & N/A \\
Type C & 0 & N/A & 2 & $375.00 \pm 530.33$ & N/A \\
Type D & 1 & $0.00 \pm 0$ & 1 & $0.00 \pm 0$ & N/A \\
Type B + C & 4 & $137.50 \pm 160.08$ & 2 & $125.00 \pm 176.78$ & 0.941 \\
Type B + D & 3 & $100.00 \pm 173.21$ & 3 & $0.00 \pm 0$ & 0.423 \\
Type C + D & 2 & $25.00 \pm 35.36$ & 2 & $150.00 \pm 212.13$ & 0.556 \\
Type B + C + D & 15 & $126.67 \pm 232.12$ & 16 & $453.13 \pm 610.05$ & 0.061 \\
All PD patients & 34 & $147.06 \pm 224.94$ & 29 & $313.79 \pm 495.68$ & 0.103 \\
\hline
\end{tabular}

Values are given as the mean \pm SD or number of females $/$ males. N/A $=$ Not available.

accounted for $66.5 \%$ of all patients. Table 2 shows the number of patients in each group and the mean levodopa dosage uptake. The mean levodopa dosage uptake was smallest in type D.

In the subgroup analysis, comparing the mean daily levodopa intake of males and females, there were no significant differences. However, there was weak evidence supporting the conclusion that females may take less levodopa than males in the type $B+C+D$ group $(p=$ 0.06 ; table 3).

\section{Discussion}

Different methods have been suggested for dividing PD patients into subtypes [2]; however, no consistent classification system has been adopted. In TCM, patients are grouped into different constitutional types based on their symptoms as described and assessed by TCM. This guides specific treatment for each patient. In this study, patients were divided into four constitutions by the TCM-CSC, and most of the PD patients (74\%) had more than one type of TCM constitution.

\section{TCM Constitution}

About 74\% of PD patients had more than one type of TCM constitution. This is close to reality, as PD is a complex disease with mixed motor and non-motor symptoms. Based on the TCM theory, the pathogenesis of PD is 'deficiency of origin' (本虛) and 'excess of superficiality' 
(標實). Clinically, PD patients have to be treated by a mixed formula with both medicines for 'strengthening body resistance' (扶正) and 'dispel pathogenic factors' (祛邪). Hence, it may not be possible to divide PD patients into a single constitution. In other words, a subtype defined as mixture of two or more constitutions is much closer to reality.

PD patients are mostly elderly people. Commonly, they may take other kinds of medicines like antihypertensive or anticholesteremic drugs in addition to anti-PD medicines. Their TCM constitution could be affected by these medicines, especially if they have been taking them for a long time. This may also be a reason why most of them may not have a single but a mixed constitution. This result is similar to previous research on this topic [13].

\section{Mean Levodopa Dosage Uptake}

The mean levodopa dosage uptake was smallest in type D (stasis constitution). However, the sample size of this trial was small, with only 1 PD patient in type B, 2 PD patients in type $\mathrm{C}$ and $2 \mathrm{PD}$ patients in type D. We cannot conclude that type D patients are having a different treatment pattern with levodopa compared to other types. Further research with a larger sample size on the treatment of different constitutions is necessary.

\section{Levodopa Intake between Genders}

There was no significant difference in the mean dosage of daily levodopa intake between females and males in each constitution. According to the TCM theory, the male is defined as 'yang' (陽) and should have much 'qi' (氣), while the female is defined as 'yin' (陰) and should have much 'blood' (血). In some sense, this may affect the treatment pattern of PD patients based on their different TCM physiology. Although there was no significant difference in the mean dosage of levodopa uptake between females and males, there may be a trend that some female PD patients (type B $+C+D)$ took less levodopa than male patients $(p=0.06)$. Further study with a larger sample size could be done to re-verify this hypothesis.

\section{Limitation}

We are aware that the sample size of this trial was small and that it was not a communitybased sample. However, the HKPDA is the largest and most well-known nonprofit organization for PD patients in Hong Kong. PD patients in different districts of Hong Kong had the same chance to participate. Hence, our results should show some truth about the actual tendency with regard to disease subtype.

The TCM-CSC is a general questionnaire to test the constitution in normal people. It is not specific to PD patients. However, there is no validated TCM questionnaire specific to PD. Based on the pattern differentiation of the TCM theory, people can be grouped into the same constitution if they have similar symptoms even if they are suffering from different diseases. As the TCM-CSC is a validated TCM constitution questionnaire used in Taiwan, it is already a good tool to reflect the body condition of PD patients at this stage.

\section{Conclusion}

PD patients cannot be divided into different TCM constitutions based on the TCM-CSC effectively. Seventy-four percent of the PD patients had more than one type of TCM constitution. Type D patients showed the lowest daily levodopa intake. There was no significant difference in the mean daily intake of levodopa between males and females in any constitution. Further research, with a larger sample size, on the treatment pattern of different TCM constitutions and on the daily levodopa intake between males and females with different TCM constitutions is recommended. 
Li et al.: Constitutional Study of Parkinson's Disease Patients Based on Traditional Chinese Medicine Pattern Differentiation

\section{Acknowledgements}

This work was supported by research grants (HMRF12132091, IRMS/12-13/1A), Mr. and Mrs. Ko Chi Ming Center for Parkinson's Disease Research from HKBU and the Dr. Ho Tzu-leung Foundation. The authors would like to thank Dr. Yan-Juan Wang from the Clinical Division, SCM, HKBU, and the HKPDA for their great support and help with this study. The authors would also like to thank Dr. Martha Dahlen for editing the English language of the manuscript.

\section{References}

1 de Lau LM, Breteler MM: Epidemiology of Parkinson's disease. Lancet Neurol 2006;5:525-535.

2 Thenganatt MA, Jankovic J: Parkinson disease subtypes. JAMA Neurol 2014;71:499-504.

3 Massano J, Bhatia KP: Clinical approach to Parkinson's disease: features, diagnosis, and principles of management. Cold Spring Harb Perspect Med 2012;2:a008870.

4 Leung H, Mok V: Parkinson's disease: aetiology, diagnosis, and management. Hong Kong Med J 2005;11:476489.

5 Simuni T, Sethi K: Nonmotor manifestations of Parkinson's disease. Ann Neurol 2008;64:S65-S80.

6 Yang H-J, Kim YE, Yun JY, et al: Identifying the clusters within nonmotor manifestations in early Parkinson's disease by using unsupervised cluster analysis. PLoS ONE 2014;9:e91906.

7 Lu H, Pan W, Wang J, Wu C, Gong F, Sun Y, Liu Y, Liu J, Liu Y, Bai Y: The current status of integrative therapies in treating Parkinson's disease in six general hospitals in Shanghai. Int J Integr Med 2013;1:17.

8 Su YC, Chen LL, Lin JD, et al: BCQ+: a body constitution questionnaire to assess Yang-Xu. 1. Establishment of a first final version through a Delphi process. Forsch Komplementmed 2008;15:327-334.

9 Chen LL, Lin JS, Lin JD, et al: BCQ+: a body constitution questionnaire to assess Yang-Xu. 2. Evaluation of reliability and validity. Forsch Komplementmed 2009;16:20-27.

10 Lin JD, Chen LL, Lin JS, et al: BCQ-: a body constitution questionnaire to assess Yin-Xu. 1. Establishment of a provisional version through a Delphi process. Forsch Komplementmed 2012;19:234-241.

11 Lin JS, Chen LL, Lin JD, et al: BCQ-: a body constitution questionnaire to assess Yin-Xu. 2. Evaluation of reliability and validity. Forsch Komplementmed 2012;19:285-292.

12 Lin JD, Lin JS, Chen LL, et al: BCQs: a body constitution questionnaire to assess stasis in traditional Chinese medicine. Eur J Integr Med 2012;4:e379-e391.

13 Todorova A, Martinez-Martin P, Schrag A, et al: Non-motor dominant profiles in Parksinson's disease: first analysis from an international naturalistic study. Poster Presentations. Mov Disord 2014;29:S1-S571. 